\title{
http://dx.doi.org/10.35381/r.k.v5i4.962
}

\section{La autogestión pública y el presupuesto de los gobiernos autónomos descentralizados municipales}

Public self-management and the budget of the municipal decentralized autonomous governments

Nancy Paola García-Cárdenas

nancy.garcia99@est.ucacue.edu.ec

Universidad Católica de Cuenca, Cuenca

Ecuador

https://orcid.org/0000-0001-9740-8042

Sonia Marlene Bonilla-Carchi

smbonilla@ucacue.edu.ec

Universidad Católica de Cuenca, Cuenca

Ecuador

https://orcid.org/0000-0002-3684-194X

Oscar Rene Calle-Masache

ocalle@ucacue.edu.ec

Universidad Católica de Cuenca, Cuenca

Ecuador

https://orcid.org/0000-0002-1605-9555

Recibido: 01 de junio de 2020

Revisado: 20 de junio de 2020

Aprobado: 02 de septiembre de 2020

Publicado: 24 de septiembre de 2020 


\title{
RESUMEN
}

El presente estudio tiene como propósito desarrollar estrategias de gestión de cobro en el GAD del cantón Azogues para el mejoramiento de la recaudación de ingresos por autogestión. fue de tipo descriptiva con diseño no experimental de campo, siendo la población constituida por funcionarios que laboran en el GAD. El rubro más representativo de los ingresos tributarios son los impuestos con el 13\%, mientras que, las tasas y contribuciones representaron el $9 \%$ En lo que concierne a la capacidad de los GAD para ejercer sus competencias se determinó que el $84 \%$ de colaboradores encuestados señalaron que la entidad está en capacidad de ejercer sus competencias establecidas en la Constitución y leyes que la rigen. La aplicación de estrategias permitirá mejorar la eficiencia en la gestión de los ingresos de la entidad, los relacionados con los impuestos municipales, tasas y contribuciones, además del fomento de la cultura tributaria en los ciudadanos.

Descriptores: Presupuesto; autogestión; gasto público; hacienda pública. (Palabras tomadas del Tesauro UNESCO).

\begin{abstract}
The purpose of this study is to develop collection management strategies in the GAD of the canton of Azogues to improve the collection of self-management income. It was descriptive with a non-experimental field design, the population being made up of officials working in the GAD. The most representative item of tax revenue is taxes with $13 \%$, while rates and contributions represented $9 \%$ Regarding the ability of GADs to exercise their powers, it was determined that $84 \%$ of employees surveyed They indicated that the entity is able to exercise its powers established in the Constitution and the laws that govern it. The application of strategies will improve the efficiency in the management of the entity's income, those related to municipal taxes, fees and contributions, as well as the promotion of a tax culture in citizens.
\end{abstract}

Descriptors: Budget; Self government; public expenditure; public finance. (Words taken from the UNESCO Thesaurus). 


\section{INTRODUCCIÓN}

El territorio del Estado ecuatoriano se encuentra organizado a nivel regional, provincial, cantonal y parroquial (áreas rurales), el órgano regulador es el Código Orgánico de Organización Territorial, Autonomía y Descentralización COOTAD (Asamblea Nacional, 2015), quien dota de personería jurídica a los Gobiernos Autónomos Descentralizados (GAD) cuya finalidad se vincula con la gestión de recursos públicos y el expendio de acuerdos según las competencias respectivas. Tal como lo afirman (Cujano, et al., 2016), la organización del gobierno ecuatoriano se asocia al proceso de descentralización, en donde se establece un rol esencial a los gobiernos locales. Parámetros que experimentan procesos de transformación política, financiera y administrativa en el territorio nacional.

Para (Castelo, et al., 2018), los GAD sustentan el presupuesto con base en la asignación presupuestaria del gobierno mediante gravámenes, contribuciones, bienes y servicios que facultan la autonomía política y financiera. No obstante, (GonzálezFontes, 2015) considera que los GAD carecen de instrumentos que articulen los elementos de planes económicos que inciden sobre el desarrollo municipal, para que posibilite al sistema administrativo asimilar el dinamismo requerido. En virtud de lo expuesto, la actividad de gobernanza de las alcandías tiene trascendencia en el Ecuador, debido a características como: la descentralización y la heterogeneidad socio económica y geográfica, por ello, es necesario la presencia de los gobiernos locales que proporcionen a la autoridad pública el conocimiento de problemas presentes en las comunidades, con esto, la resolución de forma eficiente de conflictos que satisfagan las necesidades del colectivo.

Con relación a la organización territorial, el cantón Azogues se encuentra regido por la municipalidad, de acuerdo con los lineamientos de la Constitución de la República del Ecuador, que señala mediante el artículo 238 las atribuciones de los GAD en cuanto a la autonomía política y administrativa bajo los principios de solidaridad, equidad interterritorial, integración y participación ciudadana. Sin embargo, a pesar que la Carta Suprema (Asamblea Nacional, 2008) establece el concepto de descentralización, no estipula con claridad la importancia de la asignación de 
recursos, lo que genera un proceso lleno de carencia en la práctica, que en varios casos coadyuva al incumplimiento de las metas programadas.

En consecuencia, la posibilidad de que los GAD administren los recursos es limitada, debido a que presentan información a diferentes organismos de gobierno como es el caso del Ministerio de Economía y Finanzas, Secretaría Técnica de Planificación, entre otros; se establecieron cambios en el marco normativo de los GAD para mejorar el desempeño, sin embargo, estos mecanismos no ayudan a resolver los problemas de gestión. Bajo tal contexto, el Estado pretende eliminar las deficiencias para ello otorga autonomía financiera para alcanzar el desarrollo y generar recursos para la solvencia de las necesidades locales.

La ejecución presupuestaria consiste en la relación entre lo presupuestado y lo que va en ejecución de forma efectiva, los GAD realizan este proceso con la finalidad de regular los programas y proyectos establecidos en un período, evaluados de manera trimestral por el Sistema de Información para los Gobiernos Autónomos Descentralizados (SIGAD), herramienta que permite verificar el avance de los proyectos y el uso de los recursos.

Es importante considerar que a medida que el proyecto avance, el Estado emite el presupuesto para cada GAD. A criterio de (Lozano-Calero, et al., 2019), es necesario realizar la planificación de actividades siempre y cuando se realice la verificación y control sobre las operaciones de la institución. En esta misma línea de análisis (Benalcázar-Guerrón, 2013) indica que los gobernantes y legisladores ecuatorianos muestran preocupación por los aspectos financieros relacionados con la descentralización, la autonomía financiera de los GAD y sobre los recursos que deben otorgarse.

En tal virtud, cabe indicar que la autonomía económica alude a la gestión efectiva de los fondos, mediante la independencia administrativa y el desarrollo cantonal; aspectos que encierran la importancia de la descentralización, de ahí surge la siguiente interrogante ¿Las estrategias de autogestión dirigidas a los GAD permiten mejorar la administración de recursos para un desarrollo eficiente de sus competencias? 
Bajo estos antecedentes, se plantea como problema que en el GAD del cantón Azogues a pesar de poseer una estructura organizativa funcional y participativa para la medición de los niveles de gestión en el manejo de finanzas, presentan sistemas de gestión pública deficientes que optimizan los procesos, por factores como: inobservancia de la ley, escasa planificación estratégica y la mala gestión, lo que representa una barrera para la formulación de metas y objetivos organizacionales que ayuden a cumplir los objetivos establecidos en el Plan Nacional de Desarrollo Toda una Vida (2017-2021).

En efecto, el presente estudio tiene como propósito desarrollar estrategias de gestión de cobro en el GAD del cantón Azogues para el mejoramiento de la recaudación de ingresos por autogestión. Por otra parte, es importante analizar la autonomía financiera como una exigencia para la descentralización, pues de esta forma las autoridades del GAD pueden solventar los requerimientos presentes de su gestión, mediante recursos propios y las asignaciones correspondidas por ley.

\section{Referencial teórico}

\section{La teoría de los municipios y la autogestión}

El gobierno municipal constituye el ente responsable de controlar y manejar el territorio a nivel local, por tanto, tiene autonomía en los diferentes ámbitos políticos, fiscales y administrativos, bajo los límites establecidos en la legislación. Bajo esta premisa, cabe indicar que según la (Organización de las Naciones Unidas para la Alimentación y la Agricultura (FAO), 2020) a partir de 1980 en América Latina, los municipios empezaron a asumir funciones con la división político - administrativa de los territorios. Iniciativa impulsada por la alta demanda de la ciudadanía con respecto a los servicios y la protección de los recursos de cada territorio.

Como antecedente, el proceso de autonomía municipal se consagró en el marco constitucional y legal desde 1945 y se encuentra vigente en la Constitución del Ecuador (2008). Al respecto, a partir del 2010 se aplicó el COOTAD, en donde se fija los parámetros con relación al aspecto financiero de los GAD y la autonomía económica, proporcionándole la facultad de generar recursos propios y adjudicados 
por las rentas del Estado. Por tanto, la autonomía financiera constituye el derecho facultativo de los GAD para tomar las respectivas asignaciones presupuestarias del Estado, así como los beneficios monetarios generados por la explotación del recurso natural en cuanto al territorio, de acuerdo a lo que señala la Constitución del Ecuador en su artículo 274.

Luego de la aproximación histórica de los municipios, es importante destacar algunas dificultades con respecto a la autonomía municipal, al considerar a la centralización como la fuerza del gobierno que tiende a establecer la unidad de derecho y ejecución de leyes; mientras que, la segunda busca elevar las facultades de decisión y de gestión, lo que da como resultado el denominado problema de la autonomía municipal conocida como dinámica estatal, la meta perseguida por la fuerza centrífuga y la tendencia separadora (Hernández-Aguilar, 2014).

En definitiva, el ente municipal representa la unidad política primaria de acuerdo a lo que establece la normativa legal y la responsabilidad que adquiere el alcalde frente a la ciudadanía por ser electo por voto popular para que administre la ciudad, por medio del desarrollo de las competencias en lo que concierne a la creación, recaudación e inversión de los ingresos, según lo establecido en el marco legal ecuatoriano, por tanto, la responsabilidad es de carácter vinculante a la participación ciudadana con respecto a la gestión pública.

Por otro lado, (Vaicilla-González, et al., 2020) indican que los GAD cantonales, atraviesas dificultades en el desarrollo de su gestión debido a la deficiente asignación presupuestaria, por parte del gobierno, lo que provoca el incumplimiento de metas y programas para la prestación de servicios, adquisición de bienes y ejecución de planes y programas.

A pesar de ello, se requiere tomar en cuenta los aspectos determinados en el artículo 1 de la Constitución, al señalar que el Ecuador es un Estado soberano con independencia, cuyo gobierno se encuentra descentralizado. El artículo 42 de la Constitución de la República del Ecuador (Asamblea Nacional, 2008), establece que el Estado se debe organizar bajo diversos parámetros, lo que exige que en la organización constitucional del país se contemple la existencia de autoridades y 
administraciones públicas locales con las suficientes atribuciones para solucionar las necesidades de la ciudadanía.

\section{Autonomía financiera en los gobiernos autónomos descentralizados}

Refiere a la capacidad de obtener ingresos propios y la potestad de destinarlos al fin que se estime oportuno, en el ámbito de sus competencias (Aldeguer-Cerdá, 2014). Para (Martínez \& Vayas, 2015) la autonomía refiere a la participación de los recursos propias sobre los fondos totales, mientras mayor sea este indicador, significa que la gestión en autonomía financiera de gobierno seccional es efectiva.

A nivel local el COOTAD (Registro Oficial Suplemento 303 de 19-oct-2010), en el artículo 172 menciona que son ingresos de gestión los que provienen de los impuestos, tasas y contribuciones de mejora, aquellos que provienen de la venta de bienes y servicios, de renta de inversiones y multas entre otros. El desafío y problema está en la dependencia financiera de los GAD para generar recursos propios y a partir de la Constitución (Asamblea Nacional, 2008) se incorpora el esfuerzo administrativo y fiscal con la finalidad de estimular GAD a realizar un mayor esfuerzo por manejar las finanzas de forma correcta, responsable, transparente y sostenible, lo que motiva a generar más recursos propios con la finalidad de contribuir al buen vivir de sus habitantes.

Para el ejercicio de la descentralización es necesaria la autonomía financiera, pues es ilógico pretender que las autoridades locales atiendan las necesidades del colectivo sin contar con los recursos propios para enfrentar el gasto público. Los diferentes ámbitos de los que gozan los GAD están enmarcados en el COOTAD (Registro Oficial Suplemento 303 de 19-oct-2010) en el artículo 5 en la parte pertinente a la autonomía financiera donde señala que se expresa en el derecho de los GAD para recibir de forma directa, oportuna y sin condiciones los recursos del presupuesto general del Estado y en la capacidad de generar y administrar los recursos propios según lo dispuesto en la Constitución y la ley.

Por otra parte, en los últimos años, debido al crecimiento de las necesidades colectivas, es imprescindible conocer los ingresos de los municipios vía autogestión y 
asignaciones presupuestarias del Estado; identificar la forma de reinversión del gasto para el cumplimiento de las necesidades institucionales y de la comunidad. Todos estos aspectos se reflejan en las cédulas presupuestarias de ingresos y gastos y en el estado de ejecución presupuestaria de la entidad, por lo cual, resulta necesario contar con herramientas que faciliten el control, como es el caso de la contabilidad pública o gubernamental considerada la rama que permite identificar la transparencia de la información y los registros financieros y presupuestarios del gobierno local o seccional (Sánchez, et al., 2007).

La autonomía financiera, en el ámbito ecuatoriano, se fundamenta en la Constitución (Asamblea Nacional, 2008) y en el COOTAD (Registro Oficial Suplemento 303 de 19oct-2010), en el que se establece una nueva forma de organización territorial del Estado ecuatoriano, el mismo está conformado en regiones, provincias, cantones y parroquias rurales con regímenes especiales. En el plano de la institucionalidad territorial, de igual forma, se establecen nuevos niveles de gobierno como los regionales y las mancomunidades, por tanto, se deriva la necesidad de fijar competencias que serán ejercidas como exclusivas y concurrentes por cada GAD. En cuanto a la gestión y representación, el COOTAD (Registro Oficial Suplemento 303 de 19-oct-2010) por medio del artículo 28 sostiene que cada circunscripción territorial conlleva un GAD respectivo para promocionar, desarrollar y garantizar los principios del buen vivir a través de las competencias.

Con base en lo expuesto, (Madrigal-Delgado, et al., 2018) afirman que la autonomía financiera alude a la capacidad de los gobiernos seccionales para obtener fondos propios en la cantidad requerida para cubrir las necesidades de gasto; dicho de otra forma, representa la cantidad de ingresos públicos y la decisión sobre el empleo de los mismos. 


\section{Método contable del devengado y la implementación de normativa contable internacional en el sector público}

En este sentido, (Machado-Guamantario, et al., 2020) manifiestan que el Ecuador utiliza el principio de devengado en la contabilidad gubernamental para todas las empresas que forman el sector público no financiero, para ello se acogen Sistema Nacional de Finanzas Públicas (SNFP) encargado de dictar las directrices de los procesos que deben seguir dichas entidades.

Esta etapa ocurre posterior a la etapa del compromiso, corresponde a la modificación cualitativa y cuantitativa de la composición del patrimonio, origina el pago de la transacción por el compromiso efectuado, en esta fase surge la obligación donde se procede a realizar el registro contable para dar paso a la afectación presupuestaria, en el sector público se maneja el reconocimiento de hechos económicos a las diferentes transacciones $u$ operaciones que se realizan por el registro de compromiso y el devengado de los ingresos o egresos que deben percibir y pagar los entes públicos (Medina \& Morocho, 2015).

Por otra parte, el Código orgánico de planificación y finanzas públicas, COPFP (Registro Oficial Suplemento 306 de 22-oct.-2010), en el artículo 154 establece que el registro contable y de ingresos y egresos serán de acuerdo al principio de devengado, mediante el reconocimiento y registro de un ingreso o gasto donde el período contable hace referencia a la fecha donde se realiza el desembolso o el cobro una vez que ocurra el hecho económico.

\section{Presupuesto del sector público financiero}

El presupuesto es una norma que se asigna a la institución y afecta a todas sus etapas, en el sector público los presupuestos se rigen por algunos principios como; la universalidad en la que se anotan la totalidad de los ingresos y los gastos que tenga la entidad sin haber compensaciones ni disminuciones, la unidad hace referencia a que se debe presentar un solo documento por entidad, la programación manifiesta que se debe anotar todos los ingresos que la institución necesite para el cumplimiento de los objetivos y metas trazadas, la eficiencia es la asignación de los recursos 
económicos que se le da a la institución con el fin de cumplir los costos al menor valor pero sin perder la calidad del mismo y la eficacia manifiesta que se deberá contribuir para que todas las metas y objetivos trazados en la etapa de la programación sea eficiente en el manejo de los recursos (Medina \& Morocho, 2015).

Se ha definido al presupuesto del sector público financiero como la apreciación financiera anticipada con el propósito de establecer un programa de egresos e ingresos anuales (Rodríguez-Pérez, et al., 2015). Por lo expuesto, los municipios manejan sus actividades a través del sistema del Ministerio de Finanzas en el artículo 82 del Código Orgánico de Planificación y Finanzas Públicas se entiende como componentes del SINFIP al conjunto de procesos necesarios para la organización y gestión del mismo y son la política y programación fiscal, ingresos, presupuesto, endeudamiento público, contabilidad gubernamental y tesorería (Asamblea Nacional, 2010).

Con respecto al proceso presupuestario integra la información para la toma de decisiones, bajo este contexto, es necesario estructurar un proceso capaz de integrar diferente información del desempeño, en este sentido, es preciso analizar el ciclo presupuestario del Ecuador regido por el (Ministerio de Economía y Finanzas, 2020) el mismo está compuesto por seis fases tal como se muestra en la figura 1.

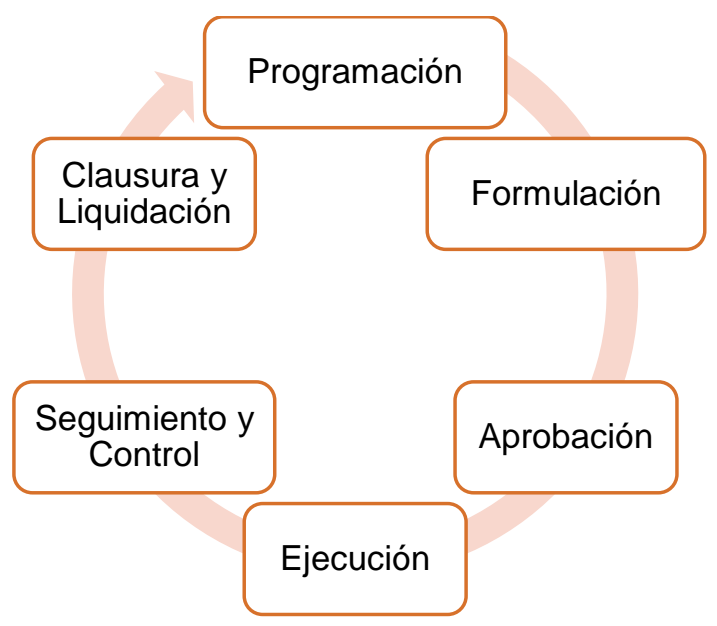

Figura 1. Ciclo presupuestario.

Fuente: Ministerio de Economía y Finanzas (2020). 
En la etapa de la programación se definen los planes, programas y actividades que se van a incorporar en el presupuesto, adicional se identifican las metas y los resultados esperados. La fase de formulación corresponde a la elaboración de las proformas que expresan los resultados de la programación, con los lineamientos de los catálogos y clasificadores presupuestarios.

Por su parte, la fase de aprobación consiste en el consentimiento del presupuesto general del Estado, deberá ser realizada bajo los términos establecidos en la Constitución. La ejecución hace referencia al conjunto de acciones destinadas al consumo de los recursos humanos, materiales y financieros asignados con la finalidad de obtener bienes, servicios y obras de calidad y oportunidad.

La fase de seguimiento comprende la medición de los resultados físicos y financieros obtenidos del análisis de las variaciones y la determinación de las causas. Por último, la clausura y liquidación establece que serán liquidados el 31 de diciembre de cada año, después de esta fecha no se podrán contraer compromisos ni obligaciones de ninguna naturaleza que afecte el presupuesto clausurado (Ministerio de Economía y Finanzas, 2020).

\section{MÉTODO}

La investigación fue de tipo descriptiva con diseño no experimental de campo, siendo la población constituida por funcionarios que laboran en el GAD, para efectos de la muestra se consideró a los 19 trabajadores del área financiera del GADMA por ser los que están más involucrados con el proceso presupuestario y de autogestión. Además, en la investigación se aplicaron varias técnicas como la revisión bibliográficadocumental para la elaboración del apartado teórico y la técnica de observación que permitió analizar el fenómeno en el curso normal.

Se recopilaron los datos mediante encuesta y cuestionario politómico de alternativas dicotómicas y escala de Likert, con la finalidad de amplificar la posibilidad de respuestas por parte de la muestra poblacional, de ese modo, se procesó la información en función de la estadística descriptiva. 


\section{RESULTADOS}

Comprende la fase de diagnóstico para ello se efectuó la encuesta al director financiero y jefes departamentales quienes conocen del manejo administrativo y contable del GAD Municipal. Desde esta perspectiva, la investigación se orientó en la autogestión pública y el presupuesto de los gobiernos autónomos descentralizados municipales, es así que la tabla 1 muestra los resultados de la aplicación del cuestionario:

\section{Tabla 1.}

Encuesta dirigida al personal del GAD de Azogues.

\begin{tabular}{|c|c|c|}
\hline Interrogante & Si & No \\
\hline La municipalidad de Azogues posee un modelo de gestión & $100 \%$ & \\
\hline $\begin{array}{l}\text { La municipalidad realiza indicadores de eficiencia en los } \\
\text { departamentos contables financieros }\end{array}$ & $100 \%$ & \\
\hline $\begin{array}{l}\text { La gestión pública de la municipalidad es eficiente al momento de } \\
\text { financiar el presupuesto de la institución }\end{array}$ & $100 \%$ & \\
\hline $\begin{array}{l}\text { El plan institucional tiene concordancia con los objetivos del plan } \\
\text { nacional de desarrollo toda una vida }\end{array}$ & $100 \%$ & \\
\hline $\begin{array}{l}\text { El presupuesto de la institución se financia mediante reformas y } \\
\text { ordenanzas }\end{array}$ & $100 \%$ & \\
\hline $\begin{array}{l}\text { La institución realiza procedimientos de control de ingresos de } \\
\text { autogestión }\end{array}$ & $100 \%$ & \\
\hline $\begin{array}{l}\text { Tanto la Constitución del país y el COOTAD establecen el sistema } \\
\text { nacional de competencia para los diferentes niveles de gobierno }\end{array}$ & $84 \%$ & $16 \%$ \\
\hline $\begin{array}{l}\text { El personal de la institución es evaluado con la finalidad de determinar } \\
\text { el grado de conocimiento de las leyes }\end{array}$ & $100 \%$ & \\
\hline
\end{tabular}

De acuerdo con el análisis de resultados, se observa en el grupo poblacional de estudio, que la totalidad (100\%) señalaron que la municipalidad de Azogues posee un modelo de gestión que le permite evaluar la situación financiera de la entidad, dato importante ya que de este modo se puede contar con información importante para el desarrollo de las actividades del municipio.

En cuanto a los indicadores se observa que el $100 \%$ de colaboradores encuestados del GADMA indicaron que si realizan este tipo de evaluaciones en 
los departamentos contables y financieros que les permiten conocer la efectividad de las operaciones realizadas en el área.

Un aspecto importante del GAD es la observación y cumplimiento de los objetivos establecidos en el Plan Nacional de Desarrollo 2017-2021 Toda una vida, con respecto a este tema al evaluar el cumplimiento de los objetivos el $100 \%$ de colaboradores encuestados indicaron que en el plan está establecido dichos objetivos, lo que genera resultados positivos en la gestión del GAD.

El 100\% de personal encuestado señala que los ingresos se generan mediante ordenanzas y se encuentran en concordancia con lo establecido en el código, que busca proporcionar las directrices a los diferentes niveles de organización territorial del país.

Por su parte, los funcionarios del GADMA han sido evaluados en el conocimiento de leyes y reglamentos que rigen a la institución, mediante capacitaciones, evaluaciones y monitoreo en el marco de la ley de competencias. En lo que concierne a lo estipulado en el COOTAD, los municipios están en la capacidad de ejercer ciertas competencias relacionadas con la prestación de servicios públicos, fomentar las actividades productivas, gestión territorial entre otras, de acuerdo a las respuestas de los colaboradores encuestados el $84 \%$ indicaron que el GAD está en la capacidad de asumir dichas competencias, mientras que el $16 \%$ señalaron que la entidad no está en la capacidad de ejercer dichas atribuciones.

Con relación al presupuesto de la entidad el $90 \%$ de personal encuestado señala que el GAD posee superávit, mientras que el $5 \%$ indicó que posee déficit. De acuerdo a los colaboradores el GAD para el desarrollo de sus funciones se financia mediante ingresos por; plusvalía, catastros, impuestos de alcabalas y patentes.

Por otro lado, con respecto a los ingresos de autogestión el 95\% de personal encuestado indicó que el $10 \%$ del presupuesto se financia con ingresos de autogestión. Mientras que, el 5\% manifestó que no existe autogestión en la GAD del cantón Azogues. Mediante la revisión teórica, la autonomía estaría supeditada a la normativa vigente y a la participación ciudadana, además el 
COOTAD, prevé la posibilidad de generar nuevos recursos como la emisión de bonos municipales, acción que, no se ha realizado en la entidad sea por la falta de conocimiento o por la ausencia de un equipo de personas calificadas para optar por esta alternativa.

Se debe considerar que las herramientas de financiamiento de los GAD y los distintos niveles de gobierno, se encuentran financiados mediante partidas presupuestarias que asigna el Estado y los ingresos de autogestión, bajo este contexto, es importante crear mecanismos que permitan cumplir con la ley y ser eficientes en el desarrollo de las actividades, la tabla 2 muestra la estructura total de los ingresos corrientes, de capital y de financiamiento.

\section{Tabla 2.}

Ingresos de GAD del cantón Azogues 2017.

\begin{tabular}{llrr}
\hline Código & \multicolumn{1}{c}{ Descripción } & \multicolumn{1}{c}{ Total } & \multicolumn{1}{c}{ Porcentaje } \\
\hline 1 & Ingresos corrientes & $\$ 6,880,694.89$ & $30 \%$ \\
1.1 & Impuestos & $\$ 2,976,704.06$ & $13 \%$ \\
1.3 & Tasas y contribuciones & $\$ 1,934,702.13$ & $9 \%$ \\
1.4 & Venta de bienes y servicios & $\$ 3,830.00$ & $0.02 \%$ \\
1.7 & Rentas de inversiones y multas & $\$ 231,629.61$ & $1 \%$ \\
1.8 & Transferencias y donaciones & $\$ 1,483,714.37$ & \\
1.9 & corrientes & $\$ 250,114.72$ & $7 \%$ \\
2 & Otros ingresos & $\$ 7,689,814.58$ & $1 \%$ \\
2.8 & Ingresos de capital & Transferencias y donaciones de capital & $34 \%$ \\
3 & e inversión & $\$ 7,689,814.58$ & \\
3.6 & Ingresos de financiamiento & $\$ 8,168,838.16$ & $34 \%$ \\
3.7 & Financiamiento público & $\$ 5,923,773.54$ & $36 \%$ \\
& Financiamiento interno & $\$ 2,245,064.62$ & $26 \%$ \\
& Total & $\$ 22,739,347.62$ & $10 \%$ \\
\hline
\end{tabular}

Fuente: GAD cantón Azogues (2017).

Con respecto a los ingresos corrientes durante el año 2017 representó el 30\% del total de ingresos, por otro lado, los ingresos de capital provenientes de la asignación del Estado representan el $34 \%$ del total y los ingresos de financiamiento reflejan la mayor fuente de ingresos durante este período con el $36 \%$ del total de los ingresos. 
El rubro más representativo de los ingresos tributarios son los impuestos con el $13 \%$, mientras que, las tasas y contribuciones representaron el $9 \%$. Con respecto a los ingresos de financiamiento la entidad recurrió en gran medida al financiamiento público pues representa el $26 \%$ del total y el financiamiento interno fue del $10 \%$, se debe destacar que la entidad recibió asignaciones del gobierno por $\$ 7,689,814.58$ millones que representa el $34 \%$ del total, lo que demuestra que la entidad tiene que implementar estrategias que le permita mejorar la recaudación corriente, ya que, está por debajo del financiamiento en $\$ 1,288,143.27$ millones de dólares.

De acuerdo a los gastos el GAD del cantón Azogues posee en su estructura los gastos corrientes, de inversión y de capital, la tabla 3 muestra que los gastos corrientes reflejan el $94 \%$ del total seguido de los gastos de capital con el $5 \%$ y en último lugar los gastos de inversión con el 0,40\%.

Tabla 3.

Gastos de GAD del cantón Azogues (2017).

\begin{tabular}{llrr}
\hline \multicolumn{1}{c}{ Código } & \multicolumn{1}{c}{ Descripción } & \multicolumn{1}{c}{ Total } & \multicolumn{1}{c}{ Porcentaje } \\
\hline 5 & Gastos corrientes & $\$ 3,801,145.65$ & $94 \%$ \\
5.1 & Gastos en personal & $\$ 2,039,360.65$ & $51 \%$ \\
5.3 & Bienes y Servicios de & $\$ 1,741,785.00$ & $43 \%$ \\
5.7 & consumo & $\$ 20,000.00$ & $0,50 \%$ \\
7 & Otros gastos & $\$ 16,000.00$ & $0,40 \%$ \\
7.3 & Gastos de inversión & $\$ 16,000.00$ & $0,40 \%$ \\
8 & Bienes y Servicios de & $\$ 209,500.00$ & $5 \%$ \\
8.4 & consumo costos de capital & $\$ 209,500.00$ & $5 \%$ \\
& Activos de larga duración & $\$ 4,026,645.65$ & $100 \%$ \\
\hline
\end{tabular}

Fuente: GAD cantón Azogues (2017).

Del gasto corriente el rubro principal es el personal con el $76 \%$ seguido de los aportes a la seguridad social con el $12 \%$ del total de gastos. Los gastos de bienes y servicios más representativos son los servicios generales con el $42 \%$ conformado por la movilización, publicidad, vigilancia y aseo. 


\section{PROPUESTA}

A partir de los resultados obtenidos, se considera pertinente desarrollar estrategias de gestión de cobro en el GAD del cantón Azogues para el mejoramiento de la recaudación de ingresos por autogestión. Para ello, es necesario implementar acciones que ayuden a generar fuentes de ingresos propios, que le permita convertirse en una institución independiente en el desarrollo y administración de sus actividades, para así, no depender de la asignación presupuestaria que otorga el Estado, en consecuencia, se plantean cuatro fases para el desarrollo de la propuesta tal como indica la figura 2.

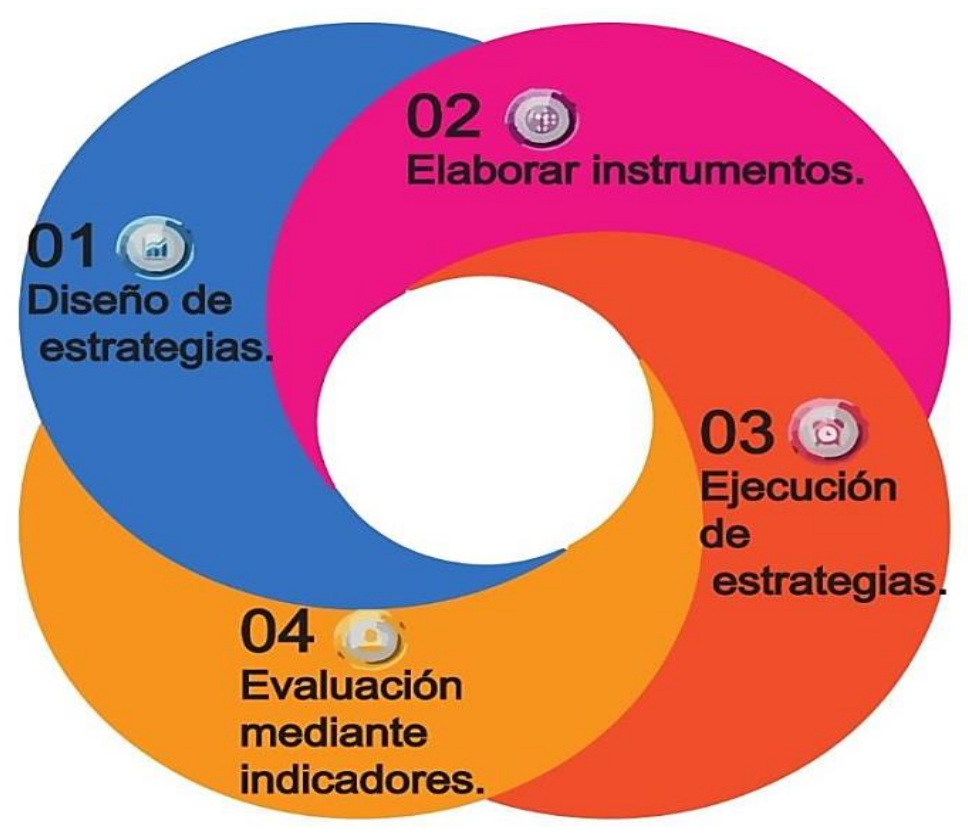

Figura 2. Propuesta de estrategias para la formulación presupuestaria del GAD municipal del cantón Azogues

\section{Objetivo de la propuesta}

Desarrollar estrategias oportunas para la adecuada gestión de cobro de los ingresos de autogestión en el GAD del cantón Azogues para el cumplimiento de los fines municipales. Se debe considerar que la recaudación de los ingresos de autogestión debe convertirse en el mecanismo más utilizado para financiar los gastos en la institución, relacionados con la ejecución de obras de infraestructura 
y mejoramiento en los servicios básicos que requiere la sociedad. En lo concerniente a los ingresos de autogestión mediante el análisis del presupuesto del GAD municipal se encontró que la entidad posee ingresos por diferentes fuentes tal como se muestra en la tabla 4:

\section{Tabla 4.}

Ingresos de autogestión del GAD.

Ingresos tributarios

Impuestos sobre la renta, utilidades y ganancias

Impuestos sobre la propiedad

Impuestos al consumo de bienes y servicios

Tasas y contribuciones

Tasas generales

Control y vigilancia municipal

Contribuciones

Venta de bienes y servicios

Fuente: GAD cantón Azogues (2017).

\section{Diseño de estrategias para el ciclo presupuestario}

El primer punto consiste en determinar estrategias para mejorar el ciclo presupuestario, para ello se observó lo que dicta la normativa Constitucional y demás leyes que rigen al GAD de Azogues, para generar cambios necesarios en los procesos de la entidad que favorezcan al cumplimiento de los objetivos. El ciclo presupuestario comprende el inicio y final de las actividades del GAD, se da inicio con la programación y el final la clausura y liquidación, sin embargo, el ciclo tiene varias identificadas en la tabla 5 : 
Tabla 5.

Fases del ciclo presupuestario.

\begin{tabular}{|c|c|c|}
\hline Fase & Descripción & Normativa aplicable \\
\hline Programación & $\begin{array}{l}\text { Se definen los planes, programas, } \\
\text { proyectos y actividades del presupuesto. }\end{array}$ & Finanzas públicas \\
\hline Formulación & $\begin{array}{l}\text { Se elaborar las proformas con los } \\
\text { resultados de la programación del } \\
\text { presupuesto. }\end{array}$ & $\begin{array}{lr}\text { Sistema } & \text { Nacional de } \\
\text { Finanzas } & \text { Públicas } \\
\text { (SINFIP) } & \end{array}$ \\
\hline Aprobación & $\begin{array}{l}\text { El plazo de aprobación comprende el año } \\
\text { donde se posesiona la máxima autoridad. }\end{array}$ & $\begin{array}{l}\text { Constitución } \\
\text { Ecuador }\end{array}$ \\
\hline Ejecución & $\begin{array}{l}\text { Se utilizan los recursos humanos, } \\
\text { materiales y financieros para obtener } \\
\text { bienes, servicios y obras. }\end{array}$ & Finanzas públicas \\
\hline $\begin{array}{l}\text { Seguimiento } \\
\text { y evaluación }\end{array}$ & $\begin{array}{l}\text { Se realiza la medición de resultados físicos } \\
\text { y financieros obtenidos, además del análisis } \\
\text { de las variaciones y sus causas. }\end{array}$ & $\begin{array}{l}\text { Secretaría Nacional de } \\
\text { Planificación y } \\
\text { Desarrollo }\end{array}$ \\
\hline $\begin{array}{l}\text { Clausura y } \\
\text { liquidación }\end{array}$ & $\begin{array}{l}\text { Los presupuestos se liquidan al } 31 \text { de } \\
\text { diciembre de cada año, pasada esta fecha } \\
\text { no se pueden contraer compromisos ni } \\
\text { obligaciones. }\end{array}$ & Finanzas públicas \\
\hline
\end{tabular}

La tabla 6 muestra estrategias y actividades que deben ser realizadas por el personal del área financiera para mejorar el manejo presupuestario de la entidad, así como el período a ejecutarse que va desde el corto, mediano y largo plazo.

Tabla 6.

Estrategias para el manejo presupuestario del GAD.

\begin{tabular}{clc}
\hline \multicolumn{1}{c}{ Estrategia } & \multicolumn{1}{c}{ Actividad } & Tiempo \\
\hline & $\begin{array}{l}\text { Capacitar al personal contable y financiero } \\
\text { relacionado con el manejo presupuestario. }\end{array}$ & Corto plazo \\
& Evaluar el desempeño de los colaboradores & Corto plazo \\
Diseñar una agenda & $\begin{array}{l}\text { de la dirección financiera del GAD. } \\
\text { presupuestaria con la }\end{array}$ &
\end{tabular}


finalidad de gestionar y administrar de manera eficiente los recursos.
Agilitar el proceso de los depósitos de los

Corto plazo recursos de autogestión con la finalidad de cumplir con los compromisos a tiempo.

Establecer la fecha límite para la evaluación presupuestaria para conocer el cumplimiento de objetivos institucionales.

Monitorizar de manera frecuente la ejecución Corto plazo del presupuesto de las unidades ejecutoras relacionándolos con la satisfacción de los usuarios.

Mejorar la gestión del personal e incrementar los niveles de formación y capacitación.

Capacitar al personal contable y financiero y demás personal de las unidades ejecutoras plazo del presupuesto, mediante cursos y seminarios relacionados con el ciclo presupuestario.

Evaluar el desempeño del personal que labora en la dirección financiera.

Crear un sistema para el control de la información con la finalidad de prevenir irregularidades. Implementar un plan de evaluación de la ejecución de los planes y programas presupuestarios.
Generar un sistema para la información Corto plazo financiera presupuestaria entre departamentos.

Identificar los parámetros para evaluar a cada Corto plazo programa o proyecto de la entidad.

Involucrar al personal de la institución en la evaluación de la ejecución presupuestaria.

Definir y delimitar roles y procedimientos del personal involucrado en la evaluación. 
Diseño e

implementación de tableros de control.
Estandarizar los componentes y términos de referencia de acuerdo a las particularidades de cada proyecto o programa.

Difundir los resultados del plan de evaluación con respecto a la ejecución del presupuesto.

Elaborar el método de semáforos para el Mediano control de las metas establecidas en los plazo proyectos y programas del presupuesto.

Realizar intervenciones para determinar el área, proyecto o programa que se va a evaluar.

Establecer una metodología para el Efectuar indicadores de seguimiento a la gestión de presupuesto. plazo

seguimiento de los

resultados del

presupuesto.

Con base a la aplicación de las estrategias se promoverá la participación activa del personal de cada área de la entidad, mediante el análisis se determinó los involucrados que serán responsables del cumplimiento y verificación de las estrategias tal como se muestra en la figura 3 :

\begin{tabular}{|c|c|c|c|c|}
\hline $\begin{array}{c}\text { Responsables } \\
\text { de la } \\
\text { planeación }\end{array}$ & $\begin{array}{c}\text { Responsables } \\
\text { de la } \\
\text { programación }\end{array}$ & $\begin{array}{c}\text { Responsables } \\
\text { de la } \\
\text { ejecución }\end{array}$ & $\begin{array}{c}\text { Responsable } \\
\text { de la } \\
\text { evaluación }\end{array}$ & $\begin{array}{c}\text { Beneficiarios } \\
\text { de los }\end{array}$ \\
\hline
\end{tabular}

Figura 3. Responsables de la implementación de estrategias 


\section{Elaborar instrumentos de aplicación}

La aplicación de los instrumentos para evaluar las estrategias permitirá identificar las ventajas de generar cambios en el proceso administrativo y contable relacionados con el ciclo presupuestarios, para ello se plantean las siguientes y estrategias en la tabla 7.

\section{Tabla 7.}

Instrumentos de evaluación de las estrategias para el manejo presupuestario del GAD.

\begin{tabular}{lll}
\hline Estrategia & Actividad & Tiempo \\
\hline Diseñar un & Identificar el objetivo y la finalidad de la evaluación. & Mediano \\
modelo de & Establecer los aspectos que se van a evaluar para & plazo \\
evaluación de la & el caso de los planes y programas. & \\
agenda & Identificar las metas que se pretende alcanzar con & \\
presupuestaria. & la evaluación. & \\
& Plantear indicadores para la evaluación de la & \\
& agenda presupuestaria. & \\
& Realizar el análisis final de los resultados. & \\
Realizar una & Establecer conclusiones y puntos de mejora. & \\
propuesta para & necesarios para alcanzarlos. & plazo. \\
el manejo & Establecer los resultados esperados en cada plan & \\
presupuestario. & programado por la entidad. &
\end{tabular}




\section{Ejecución de estrategias}

Para la implementación de las estrategias es necesario realizar un plan de implementación mediante la identificación de ciertos parámetros o actividades que debe seguir el GAD municipal de Azogues en representación de los colaboradores del área financiera, la tabla 8 muestra cada etapa.

\section{Tabla 8.}

Etapas de ejecución de estrategias.

\begin{tabular}{ll}
\hline Contexto legal & $\begin{array}{l}\text { Plasmar en un documento interno los responsables y las } \\
\text { funciones del personal. }\end{array}$ \\
\hline $\begin{array}{l}\text { Capacitar al } \\
\text { personal }\end{array}$ & Capacitar a los colaboradores del GAD sobre las estrategias \\
$\begin{array}{l}\text { Sensibilizar al } \\
\text { personal } \\
\begin{array}{l}\text { Supervisar las } \\
\text { actividades }\end{array}\end{array}$ & $\begin{array}{l}\text { Intercambio de experiencias, ideas y rendición de cuentas } \\
\text { designado }\end{array}$
\end{tabular}

\section{Evaluación mediante indicadores}

Como último punto se propone aplicar medidas de gestión para evaluar el grado de cumplimiento de las funciones y responsabilidades de los colaboradores, para ello se establece los siguientes indicadores de efectividad de la recaudación de ingresos por autogestión: Los indicadores de gestión tienen como finalidad asegurar el cumplimiento de la recaudación tributaria mediante su medición oportuna y determinar las causas del incumplimiento para tomar acciones de mejora en la tabla 8 se muestra de forma detallada el objetivo de cada actividad y la forma de medición. 


\section{Tabla 9.}

Indicadores de las estrategias para el manejo presupuestario del GAD.

\section{GAD municipal del cantón Azogues}

\section{Evaluación de la gestión presupuestaria}

Objetivo general: Mejorar el manejo de los fondos públicos y los procesos de gestión presupuestaria en el GAD municipal de Azogues.

Indicador: Indicadores de aprendizaje y crecimiento, de desempeño, productividad, control y monitoreo.

\section{Objetivos estratégicos}

Actividad

Indicador

\begin{tabular}{|c|c|c|}
\hline $\begin{array}{l}\text { Desarrollar y } \\
\text { fortalecer las } \\
\text { competencias } \\
\text { sobre gestión } \\
\text { presupuestaria. }\end{array}$ & $\begin{array}{l}\text { Capacitar al } \\
\text { contable yersonal } \\
\text { relacionado con el manejo } \\
\text { presupuestario. }\end{array}$ & $\frac{\text { Número de capacitaciones ejecutadas }}{\text { Número de capacitación programadas }}$ \\
\hline $\begin{array}{l}\text { Medir la } \\
\text { efectividad de } \\
\text { las actividades } \\
\text { realizadas por el } \\
\text { personal } \\
\text { financiero. }\end{array}$ & $\begin{array}{l}\text { Evaluar el desempeño de } \\
\text { los colaboradores de la } \\
\text { dirección financiera del } \\
\text { GAD. }\end{array}$ & $\begin{array}{c}\text { Actividades realizadas durante el período } \\
\text { Presupuestario }\end{array}$ \\
\hline $\begin{array}{l}\text { Optimizar el } \\
\text { tiempo de } \\
\text { depósitos de los } \\
\text { fondos } \\
\text { obtenidos. }\end{array}$ & $\begin{array}{l}\text { Agilizar el proceso de los } \\
\text { depósitos de los recursos de } \\
\text { autogestión con la finalidad } \\
\text { de cumplir con los } \\
\text { compromisos a tiempo. }\end{array}$ & $\begin{array}{l}\text { Registro de las fechas de depósitos de los } \\
\text { Fondos }\end{array}$ \\
\hline $\begin{array}{l}\text { Programar los } \\
\text { procesos de } \\
\text { evaluaciones } \\
\text { presupuestarias. }\end{array}$ & $\begin{array}{l}\text { Establecer la fecha límite } \\
\text { para la evaluación } \\
\text { presupuestaria para } \\
\text { conocer el cumplimiento de } \\
\text { objetivos institucionales. }\end{array}$ & $\frac{\text { Número de evaluaciones ejecutadas }}{\text { Número de evaluaciones programadas }}$ \\
\hline $\begin{array}{l}\text { Mantener } \\
\text { niveles } \\
\text { adecuados de } \\
\text { satisfacción de } \\
\text { los usuarios. }\end{array}$ & $\begin{array}{l}\text { Monitorizar la ejecución del } \\
\text { presupuesto de las } \\
\text { unidades ejecutoras } \\
\text { relacionándolos con la } \\
\text { satisfacción de los usuarios. }\end{array}$ & Número de monitoreo efectuados \\
\hline $\begin{array}{l}\text { Mejorar el } \\
\text { conocimiento y } \\
\text { las habilidades } \\
\text { prácticas del } \\
\text { personal sobre } \\
\text { la ejecución } \\
\text { presupuestaria. }\end{array}$ & $\begin{array}{l}\text { Capacitar al personal } \\
\text { contable y financiero y } \\
\text { demás personal de las } \\
\text { unidades ejecutoras del } \\
\text { presupuesto, mediante } \\
\text { cursos y seminarios }\end{array}$ & $\frac{\text { Número de capacitaciones ejecutadas }}{\text { Número de capacitaciones programadas }}$ \\
\hline
\end{tabular}




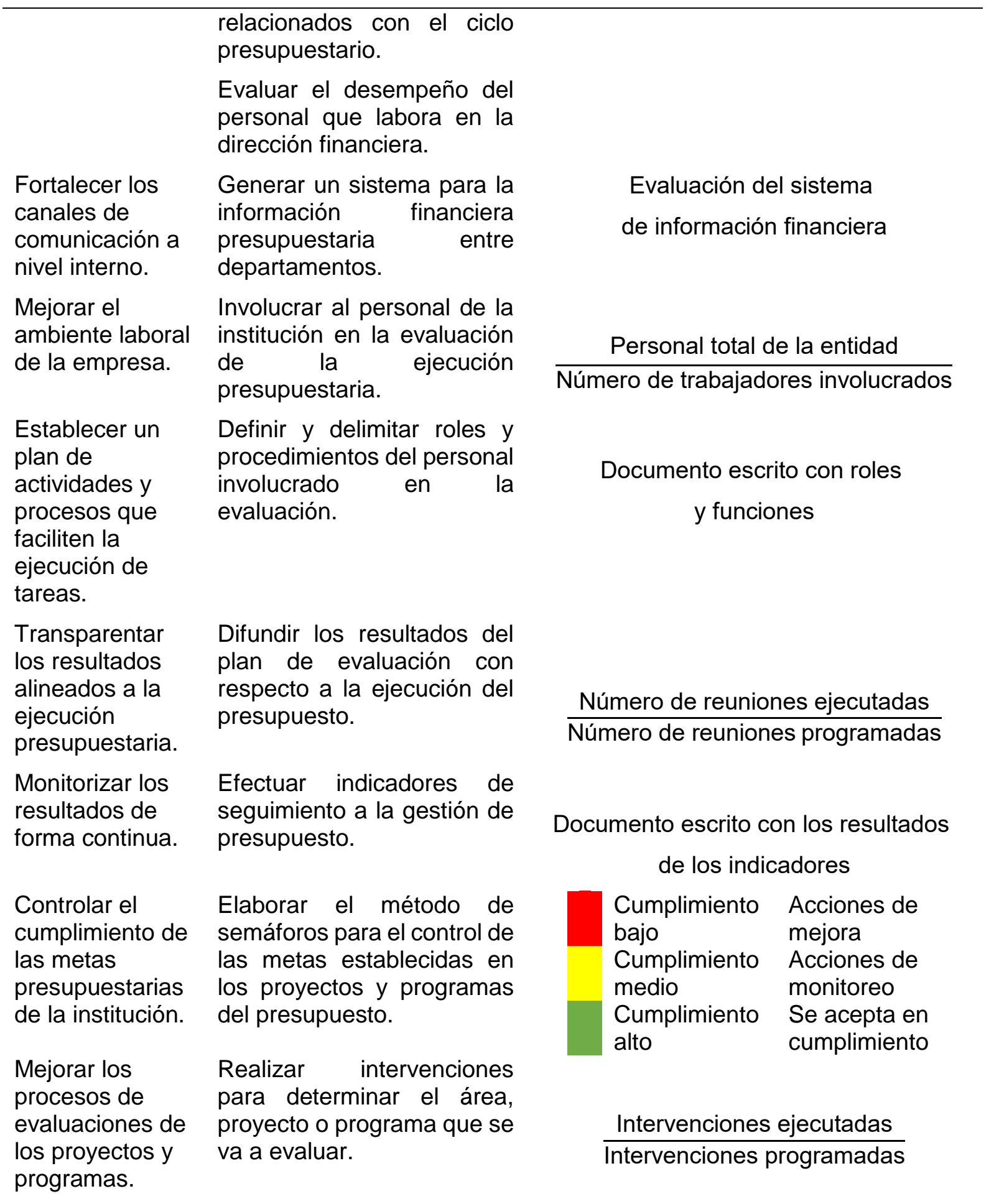




\section{DISCUSIÓN Y CONCLUSIONES}

El presupuesto público comprende un mecanismo importante para alcanzar los objetivos de la población, con respecto a los GAD deben realizar su presupuesto de forma eficiente con la finalidad de asegurar el adecuado uso de los recursos públicos. Por otro lado, se determinó que el presupuesto comprende una estimación planificada, de los programas y los resultados que se esperan obtener durante un período determinado, representa la expresión de los datos cuantitativos de los objetivos y las estrategias utilizadas para alcanzarlos.

Al analizar la autonomía financiera se describe que es el eje central de las organizaciones territoriales, al respecto, (Ayala-Pasquel, et al., 2017) señalan que los GAD del Ecuador gozan de total autonomía y ninguna instancia del Estado puede interferir en la gestión que realizan en el territorio. Según el análisis del GAD municipal de Azogues se evidenció que el $30 \%$ de los ingresos totales son corrientes, estos dependen de la normativa de recaudación establecida en ordenanzas y reglamentos municipales que les faculta el COOTAD para recaudar recursos por cuenta propia. Resultados inferiores encontró (Jiménez , 2019) al evaluar la ejecución presupuestaria del GAD municipal de Santo Domingo durante el año 2016 pues representó el 24,30\% de los ingresos totales.

Los resultados de la investigación permitieron determinar que el GAD de Azogues para el cumplimiento de sus actividades sustenta los ingresos mediante gestión propia, por las transferencias del gobierno central y mediante fondos de instituciones financieras públicas, este conjunto de fondos permite a la entidad realizar los proyectos programados para el período.

La aplicación de estrategias permitirá mejorar la eficiencia en la gestión de los ingresos de la entidad, los relacionados con los impuestos municipales, tasas y contribuciones, además del fomento de la cultura tributaria en los ciudadanos. En lo que concierne a la capacidad de los GAD para ejercer sus competencias se determinó que el $84 \%$ de colaboradores encuestados señalaron que la entidad está en capacidad de ejercer sus competencias establecidas en la Constitución y leyes que la rigen.

A criterio de (Charro-Cisnero, et al., 2020) en el Ecuador las competencias de los GAD son de índole exclusiva o recurrente, es decir, que pueden ser ejercidos con otros 
niveles de gobierno. Mediante el uso de las competencias las entidades para la aplicación y desarrollo de sus procesos pueden utilizar diferentes fuentes de ingresos que proporcionen mayores ventajas a los ciudadanos.

\section{FINANCIAMIENTO}

No monetario.

\section{AGRADECIMIENTO}

A los 19 trabajadores del área financiera del GADMA involucrados con el proceso presupuestario y de autogestión; por apoyar el desarrollo de la investigación.

\section{REFERENCIAS CONSULTADAS}

Aldeguer-Cerdá, B. (2014). Autonomía Local, equilibrio presupuestario y sostenibilidad financiera. Tendencias y perspectivas contemporáneas en las relaciones de tutela financiera y control presupuestario local en España (20082014) [Local Autonomy, budget balance and financial sustainability. Contemporary trends and perspectives in the relationships of financial supervision and local budget control in Spain (2008-2014)]. Cuadernos De Gobierno Y Administración Pública, 1(2), 181-218. https://doi.org/10.5209/rev CGAP.2014.v1.n2.47541

Asamblea Nacional. (2008). Constitución del Ecuador 2008. [Constitution of Ecuador 2008]. Recuperado de https://n9.cl/hd0q

Asamblea Nacional. (2010). Código Orgánico de Planificación y Finanzas Públicas. [Organic Code of Planning and Public Finance]. Recuperado de: https://n9.cl/pgdda

Ayala-Pasquel, S., Zaruma-Ávila, M., \& Barragán-Sánchez, H. (2017). Origen y destino de recursos del presupuesto de los GADs Municipales: estudio del Cantón Morona [Origin and destination of resources from the budget of the Municipal GADs: study of the Morona Canton]. Killkana Social, 1(3), 2936. https://doi.org/10.26871/killkana social.v1i3.60

Benalcázar-Guerrón, J. (2013). La autonomía financiera municipal en el Ecuador, con especial referencia a los ingresos tributarios y a la potestad tributaria de los municipios. [Municipal financial autonomy in Ecuador, with special reference to tax revenue and to municipal tax jurisdiction]. Revista IUS, 7(32), 27-40. 
Castelo, M., Vallejo, M., Villacrés, W., \& Caiza, R. (2018). Modelo de evaluación presupuestaria para los gobiernos autónomos descentralizados municipales. [Budget evaluation model for municipal decentralized autonomous governments]. Recuperado de https://n9.cl/trwm

Charro-Cisnero, D., Midero-Mora, A., \& Heinz-Gaudri, K. (2020). Economía circular desde la competencia de áridos y petróleos en los GAD municipales del Ecuador. [Circular economy from the competition of aggregates and oils in the municipal GAD of Ecuador]. GEO Latitud, 3(1), 24-33.

Cujano, J., Guilcapi, L., \& Erazo, L. (2016). Diagnóstico de la gestión administrativa en los gobiernos autónomos descentralizados parroquiales rurales del cantón guano: un enfoque de la participación ciudadana. [Diagnosis of administrative management in rural parish decentralized autonomous governments of the guano canton: an approach to citizen participation]. Recuperado de https://n9.cl/txbh8

GAD Cantón Azogues (2017). Ingresos de GAD del cantón Azogues 2017. [GAD revenues of the canton Azogues 2017]. Recuperado de https://n9.cl/slvf

González-Fontes, R. (2015). Implementación de la estrategia de desarrollo municipal, eje articulador del sistema de gestión del desarrollo local. [Implementation of a Strategy for Municipal Development: Linchpin of Management System in Local Development]. Retos de la Dirección, 9(1), 121-144.

Hernández-Aguilar, O. (2014). La autonomía municipal en el constitucionalismo latinoamericano: realidad y perspectivas en el caso de Cuba. [The municipal autonomy in the latin american Constitucionalism: reality and perspectives In the case of Cuba]. Cuestiones Constitucionales Revista Mexicana de Derecho Constitucional, 1(30). http://dx.doi.org/10.1016/S1405-9193(14)70460-3

Jiménez, P. (2019). Análisis de la ejecución presupuestaria en el gobierno autónomo descentralizado municipal de Santo Domingo y su desarrollo económico durante los años 2015 y 2016. [Analysis of budget execution in the Santo Domingo municipal decentralized autonomous government and its economic development during 2015 and 2016] Recuperado de https://n9.cl/qma4

Lozano-Calero, G. M., Narváez-Zurita, C. I., Erazo-Álvarez, J. C., \& Montero-Cobo, M. A. (2019). Procedimientos de control y registro contable bajo NIC SP para los activos del Gobierno Autónomo Descentralizado Parroquial de Fundochamba de la provincia de Loja. [Accounting control and registration procedures under NIC SP for the assets of the Autonomous Decentralized Parish Government of Fundochamba in the province of Loja]. Visionario Digital, 3(2.1.), 388-419. https://doi.org/10.33262/visionariodigital.v3i2.1.582 
Machado-Guamantario, J., Narváez-Zurita, C., \& Erazo-Álvarez, J. (2020). Evaluación y medición del principio del devengado en el sector público no financiero del Ecuador. [Evaluation and measurement of the accrual principle in the nonfinancial public sector of Ecuador]. Revista Arbitrada Interdisciplinaria Koinonía, 5(10), 160-194. http://dx.doi.org/10.35381/r.k.v5i10.692

Madrigal-Delgado, G, Camacho-Castro, C, González-Franco, R, \& Bueno-Cevada, L. (2018). Desempeño fiscal municipal del estado de Sinaloa: Propuesta de índice sintético para evaluaciones fiscales. [Municipal fiscal performance of the State of Sinaloa: Proposal of synthetic index for fiscal evaluations]. Economía, sociedad y territorio, 18(57), 359-396. https://doi.org/10.22136/est20181175

Martínez, J., \& Vayas, T. (2015). Indicadores de gestión del GAD Municipalidad de Patate. [Management indicators of the GAD Municipality of Patate]. Boletín De Coyuntura, 0(6), 9-12. http://dx.doi.org/10.31164/bcoyu.6.2015.573

Medina, W., \& Morocho, Z. (2015). Contabilidad Gubernamental I. [Government Accounting I]. Recuperado de http://repositorio.utmachala.edu.ec/handle/48000/6790

Ministerio de Economía y Finanzas. (2020). El ciclo presupuestario. [The budget cycle]. Recuperado de https://n9.cl/17uz

Organización de las Naciones Unidas para la Alimentación y la Agricultura (FAO). The role of municipal governments. Recuperado de https://n9.cl/dhzn8

Registro Oficial Suplemento 303 de 19-oct-2010. Código Orgánico Organización Territorial Autonomía Descentralización, COOTAD. [Organic Code Territorial Organization Autonomy Decentralization, COOTAD]. Recuperado de https://n9.cl/d9vg

Registro Oficial Suplemento 306 de 22-oct.-2010. Código orgánico de planificación y finanzas públicas, COPFP. [Organic planning and finance code public, COPFP]. Recuperado de https://n9.cl/4csc

Rodríguez-Pérez, H, Fernández-Andrés, A, \& De-Dios-Martínez, A. (2015). Sobre el análisis de la gestión presupuestaria con enfoque de riesgos. [Analysis of budgetary management with risks approach]. Retos de la Dirección, 9(1), 2344.

Sánchez, J., Pincay, D., Guevara, F., \& Calle, J. (2007). Gestión y el Devengo. Contabilidad Pública. [Management and Accrual. Public accounting] Recuperado de https://n9.cl/rqpnh. 
Nancy Paola Garcia-Cárdenas; Sonia Marlene Bonilla-Carchi; Oscar Rene Calle-Masache

Vaicilla-González, M., Narváez-Zurita, C., Erazo-Álvarez, J., \& Torres-Palacios, M. (2020). Transparencia y efectividad en la ejecución presupuestaria y contratación pública en los gobiernos cantonales. [Transparency and effectiveness in budget execution and public procurement in cantonal governments]. Revista Arbitrada Interdisciplinaria Koinonía, 5(10), 774-805. 